\title{
Role of Neutrophil-to-Lymphocyte Ratio as a Predictor of Systemic Inflammatory Response Syndrome in Blunt Abdominal Trauma Following Emergency Laparotomy in Sanglah General Hospital, Denpasar
}

\author{
I Made Dwi Purnamantara(D), I Ketut Wiargitha, Tjok Gede Bagus Mahadewa* \\ Department of Surgery, Faculty of Medicine, Udayana University, Sanglah General Hospital, Denpasar, Bali, Indonesia
}

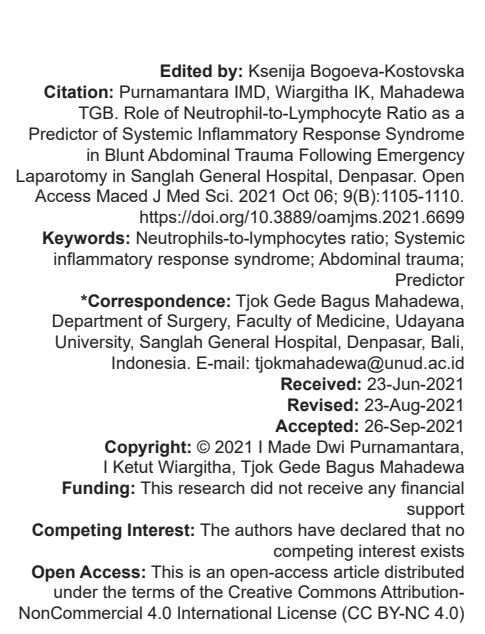

Abstract

BACKGROUND: Abdominal trauma, both blunt and penetrating abdominal trauma, is the third most common trauma, which is about $10 \%$ after head trauma $(30 \%)$ and chest trauma $(20 \%)$. Trauma has an influence on the balance of the immune system. The excessive inflammatory response can trigger systemic inflammatory response syndrome (SIRS). The ratio of neutrophils to lymphocytes acts as a marker of the inflammatory response in both surgical and non-surgical patients.

AIM: The purpose of the study was to predict the incidence of SIRS using neutrophils-to-lymphocytes ratio (NLR) in patients with blunt abdominal trauma undergoing emergency laparotomy.

METHODS: This study is an observational analytic study with a prospective design which was carried out at the emergency room, intensive care unit, Sanglah Hospital, Denpasar, Surgery for 1 year from January 2019 to December 2019, involving 59 research subjects with the consecutive sampling method who is met the inclusion and exclusion criteria.

RESULTS: The area under the curve value obtained an NLR sensitivity of $87.1 \%$ with a cutoff point of 5.25 . In the path analysis, it was found that NLR was the dominant factor and had a positive effect of $43.9 \%(p<0.001)$ on SIRS. CONCLUSION: NLR is expected to be used as a reference to predict the incidence of SIRS in blunt abdominal trauma following laparotomy emergency.

\section{Background}

Abdominal trauma, both blunt and penetrating trauma, is the third most common trauma, which is about $10 \%$ after head trauma (30\%) and chest trauma (20\%) [1]. Management of trauma patients, especially those related to preventing blood loss and coagulopathy, significantly reduced the mortality ratio directly related to trauma. However, secondary complications including acute respiratory distress syndrome, multiple organ failure, and nosocomial infections are significant causes of death in hospitalized trauma patients [2]. Trauma counts as one of the many factors related to immune system function changes. The development of these changes basically caused by changes in one particular cells, termed as neutrophils, which have an important role in development of immune system functions. Trauma has an influence on the balance of the immune system between the pro-inflammatory and anti-inflammatory systems [2]. The imbalance in these two systems affects the post-trauma outcome.
The excessive inflammatory response can trigger the systemic inflammatory response syndrome (SIRS) [3]. The neutrophil-lymphocyte ratio (NLR) is a reliable, fast, and simple method of evaluating the extent of the stress and inflammatory response. The NLR in various studies is a reliable predictor of sepsis, but the lack of research data on the NLR in abdominal trauma patients requires research on this matter.

\section{Methods}

This study is an observational analytic study with a prospective design to determine the predictive value of the incidence of SIRS from the NLR in blunt abdominal trauma performed emergency laparotomy and diagnostic tests to determine the sensitivity, specificity, and accuracy of the NLR. The research was conducted at the emergency room (ER), intensive care unit (ICU), Sanglah General Hospital Surgery, 
Denpasar. This research was conducted for 1 year from January 2019 to December 2019. Sampling as research subjects was carried out by a consecutive sampling method involving 59 research subjects who met the inclusion and exclusion criteria.

\section{AQ6 Results}

The characteristics of the respondents in this study are presented in the form of a single distribution table.

Table 1 describes the study sample characteristics. Patients age category was divided into two categories, with 36 years as cutoff point. From patients aged more than 36 years old, 23 respondents $(51.1 \%)$ have a positive SIRS and negative SIRS in 3 respondents $(21.4 \%)$ while the age category $<36$ years have 22 positive SIRS respondents $(48.9 \%)$ and 11

AQ7 respondents $(78.6 \%)$ negative SIRS with $p=0.051$. With $p>0.05$, there are no statistical difference in SIRS events between respondents below and over 36 years old.

Table 1: Characteristics of respondents based on age, gender, $\mathrm{BMI}$, glucose level, trauma status, and anion gap

\begin{tabular}{|c|c|c|c|}
\hline \multirow[t]{2}{*}{ Variable } & \multicolumn{2}{|l|}{ SIRS } & \multirow[t]{2}{*}{$\mathrm{p}$} \\
\hline & SIRS (+) & SIRS (-) & \\
\hline \multicolumn{4}{|l|}{ Age (years, \%) } \\
\hline$\geq 36$ years & $23(51.1)$ & $3(21.4)$ & \multirow[t]{2}{*}{0.051} \\
\hline$<36$ years & $22(48.9)$ & $11(78.6)$ & \\
\hline \multicolumn{4}{|l|}{ Gender (n\%) } \\
\hline Male & $32(71.1)$ & $9(64.3)$ & \multirow[t]{2}{*}{0.628} \\
\hline Female & $13(28.9)$ & $5(35.7)$ & \\
\hline \multicolumn{4}{|l|}{ BMI $\left(\mathrm{kg} / \mathrm{m}^{2}, \mathrm{n} \%\right)$} \\
\hline Abnormal $\left(<18.5\right.$ or $\left.>24.9 \mathrm{~kg} / \mathrm{m}^{2}\right)$ & $14(31.1)$ & $1(7.1)$ & \multirow[t]{2}{*}{0.072} \\
\hline Normal $\left(18.5-24.9 \mathrm{~kg} / \mathrm{m}^{2}\right)$ & 31 (68.9) & $13(92.9)$ & \\
\hline \multicolumn{4}{|l|}{ Glucose level (gr/dl, n\%) } \\
\hline$\geq 140 \mathrm{gr} / \mathrm{dl}$ & $28(62.2)$ & $4(28.6)$ & \multirow[t]{2}{*}{0.027} \\
\hline$<140 \mathrm{gr} / \mathrm{dl}$ & $17(37.8)$ & $10(71.4)$ & \\
\hline \multicolumn{4}{|l|}{ Trauma status ( $n \%)$} \\
\hline Multitrauma & $32(71.1)$ & $3(21.4)$ & \multirow[t]{2}{*}{0.001} \\
\hline Monotrauma & $13(28.9)$ & $11(78.6)$ & \\
\hline \multicolumn{4}{|l|}{ Anion gap (n\%) } \\
\hline Anion gap ( $\geq 12)$ & $35(77.8)$ & $6(42.9)$ & \multirow[t]{2}{*}{0.013} \\
\hline Anion gap $(<12)$ & $10(22.2)$ & $8(57.1)$ & \\
\hline
\end{tabular}

The gender characteristics in this study showed that 32 respondents $(71.1 \%)$ had a positive SIRS and $9(64.3 \%)$ had negative SIRS, while 13 respondents (28.9\%) got a positive SIRS and 5 respondents with negative SIRS $(35.7 \%)$ with $p=0.628$ that means gender not significantly related with SIRS.

Characteristics based on BMI, abnormal BMI with positive SIRS is 14 respondents $(31.1 \%)$ and negative SIRS 1 respondent $(7.1 \%)$ while normal BMI got positive SIRS is 31 respondents $(68.9 \%)$ and negative SIRS 13 respondents $(92.9 \%)$ with $p=0.072$.

In this study, it was found that 28 respondents with high glucose level $(62.2 \%)$ had positive SIRS while 4 respondents $(28.6 \%)$ had negative SIRS. Seventeen respondents with normal glucose level had a positive SIRS (37.8\%) and negative SIRS of 10 respondents $(71.4 \%)$ with $p=0.027$ which shows that blood sugar levels are significantly associated with SIRS.
The anion gap is significantly related with SIRS. In this study, we found that 35 respondents (77.8\%) with high anion gap had developed SIRS and 6 respondents (42.9\%) with high anion gap didn't develop SIRS. Respondents who had normal anion gap value, 10 respondents $(22.2 \%)$ had developed SIRS and 8 respondents $(57.1 \%)$ did not develop SIRS with $p=$ 0.013 .

In multiple trauma patients, 32 patients had positive SIRS $(71.1 \%)$ and negative SIRS by 3 patients $(21.4 \%)$ while non-multiple trauma patients obtained positive SIRS by $13(28.9 \%)$ and negative SIRS as many as 11 patients (78.6\%) with $p=0.001$.

NLRs sensitivity to the incidence of SIRS on blunt abdominal trauma carried out by laparotomy described in the receiver operating characteristic (ROC) curve and area under the curve (AUC) value.

NLRs sensitivity is obtained $87.1 \%$ based on AUC value. AUC value $>70 \%$ is very satisfying that means in 59 respondents carried out laparotomy obtained $87.1 \%$ or 51 people suffering from SIRS (Figure 1). Furthermore, the cutoff point value is sought based on the ROC curve coordinates processed in Microsoft Excel, the cutoff point 5.25 is then carried out by a cross-tabulation so that data are obtained such as Table 2.

Table 2: NLRs sensitivity to the incidence of SIRS on blunt abdominal trauma carried out by emergency laparotomy

\begin{tabular}{|c|c|c|c|c|c|c|c|c|}
\hline \multirow[t]{2}{*}{ NLR } & \multicolumn{2}{|l|}{ SIRS } & \multirow[t]{2}{*}{ Sensitivity } & \multirow[t]{2}{*}{ Specificity } & \multirow[t]{2}{*}{ NPP } & \multirow[t]{2}{*}{ NPN } & \multirow{2}{*}{\multicolumn{2}{|c|}{ Accuracy RR }} \\
\hline & SIRS (+) & SIRS (-) & & & & & & \\
\hline$\geq 5.25$ & $36(80 \%)$ & $3(21.4 \%)$ & $92.3 \%$ & $55 \%$ & $80 \%$ & $78 \%$ & $79.6 \%$ & 2 \\
\hline$<5.25$ & $9(20 \%)$ & $11(78.6 \%)$ & & & & & & \\
\hline
\end{tabular}

NLR: Neutrophil-lymphocyte ratio, SIRS: Systemic inflammatory response syndrome, RR. Relative risk.

Table 2 shows the NLR variable $\geq 5.25$ with positive SIRS as many as 36 respondents (80\%) and negative SIRS as many as 3 respondents $(21.4 \%)$ while NLR $<5.25$ obtained fewer incidence of positive SIRS 9 respondents (20\%) and negative SIRS 11 respondents (78.6\%). Sensitivity test results based on Table $2 \times 2$ obtained $92.3 \%$, specificity of $55 \%$, a positive predictive value of $80 \%$, and a negative predictive value of $78 \%$ with an accuracy of $79.6 \%$. Then determine the incident $1\left(I_{1}\right)$ is $\frac{a}{a+b}$ and incident $2\left(I_{2}\right)$ is $\frac{c}{c+d}$. Then determine AQ9 Relative Risk (RR) by dividing $\frac{11}{12}$. Based on $2 \times 2$ tables obtained RR 2. RR> 1 that means NLR increases the risk of the occurrence of SIRS on blunt abdominal trauma carried out by laparotomy emergency.

Multivariate analysis with categorical data using logistic regression tests. This method starts with entering all variables into the model. Logistic regression test with backward method was analyzed to measure if the test model can be used in this study. In the Omnibus table test, $p<0.05$ means that the test model can be continued because the independent variable affects the dependent variable.Hosmer and Lemeshow test was performed, and obtained $p$ value $>0.05$, stating this test 
model is fit for use. Classification table obtained $89.8 \%$ which means the test model used in predicting the independent variable to the dependent variable of $89.8 \%$.

Table 3 shows the results of the variables put together and then selected to produce the respondent's age data with a value of $p=0.414$, BMI with $p=0.118$, blood sugar levels with $p=0.946$, anion gap with $p=0.932$, and the status of trauma $p=0.059$. The end result of this analysis is obtained that NLR has the smallest $p=0.005$ compared with other variables. The value of $B$ describes that NLR is positively related to the SIRS incident. Adjusted OR 15.6 NLR is also higher than the other variables, means NLR $\geq 5.25$ increasing risk of SIRS 15.6 times in abdominal trauma patients. NLR is the most dominant risk factor in this research.

Table 3: Influence of NLR toward SIRS events by controlling variables including age, BMI, blood sugar levels, anion gap, and traumatic status

\begin{tabular}{lllll}
\hline Variable & $\mathrm{B}$ & $\mathrm{OR}$ & $\mathrm{IK} 95 \%$ & $\mathrm{p}$ \\
\hline Age & 0.82 & 2.2 & $0.31-16.74$ & 0.414 \\
BMI & 2.03 & 7.6 & $0.59-97.60$ & 0.118 \\
Glucose level & 0.07 & 1 & $0.13-8.29$ & 0.946 \\
Anion gap & -0.09 & 0.9 & $0.11-7.38$ & 0.932 \\
Trauma status & 2.06 & 7.9 & $0.92-67.62$ & 0.059 \\
NLR & 2.75 & 15.6 & $2.32-105.72$ & 0.005 \\
\hline NLR: Neutrophil-lymphocyte ratio, SIRS: Systemic inflammatory response syndrome, BMI: Body mass \\
index.
\end{tabular}

\section{Path analysis}

Path analysis is a further part of regression analysis with the aim of determining the indirect effect of the free variable in the form of an interval or ratio of the dependent variable. The stages of path analysis are conducting a linear regression test of the BMI, glucose levels, and gap anions against NLR.

Table 4: Regression analysis in all variables

\begin{tabular}{lllllll}
\hline Variable & $\mathrm{B}$ & $\mathrm{R}$ square & Beta & $\mathrm{t}$ & $\mathrm{IK} 95 \%$ & $\mathrm{p}$ \\
\hline BMl & -0.008 & 0.20 & -0.054 & -0.441 & $-0.04-0.02$ & 0.661 \\
Glucose level & -0.006 & & -0.080 & -1.547 & $-0.00-0.00$ & 0.129 \\
Anion gap & -0.015 & & -0.024 & -3.362 & $-0.29-0.00$ & 0.001 \\
\hline
\end{tabular}

Table 4 describes the influence of BMI, glucose, and anion gap against NLR. Based on the value of $B$ and beta, obtained anion gap associated with NLR with $p \leq 0.05(p=0.001)$ compared to glucose levels $(p=0.129)$ and BMI ( $p=0.661)$. The magnitude of the effect of BMI, glucose, and anion gap against NLR expressed in the value of $R$ square. This means that the three variables contributed $20 \%$ and other variables that were not included in this study caused the rest. Furthermore, the analysis was continued to find out that BMI, glucose levels, anions gap, and NLR associated with SIRS.

Table 5 describes the influence of the BMI, glucose levels, anions gaps, and NLR toward SIRS. In this study, NLR had an influence on the SIRS with $p \leq 0.001$ and glucose levels with $p=0.029$ while the BMI with $p=0.403$ and anion gap with $p=0.085$. The magnitude of the influence of the BMI variables, glucose levels, anion gap, and NLR is $34.1 \%$, means $65.9 \%$ because of the influence of other variables that are not included in this model. The next test is to determine the direct and indirect effect of the IMT variables, glucose levels, and anion gaps, and NLR to SIRS is depicted in Figure 2.

Table 5: The influence of BMI, anion gap glucose level, and NLR toward SIRS

\begin{tabular}{lllllll}
\hline Variable & $\mathrm{B}$ & $\mathrm{R}$ square & Beta & $\mathrm{T}$ & $\mathrm{IK} 95 \%$ & $\mathrm{p}$ \\
\hline BMI & 0.012 & 0.341 & -0.094 & -0.843 & $-0.041-0.017$ & 0.403 \\
Glucose level & 0.001 & & 0.249 & 2.250 & $-0.003-0.000$ & 0.029 \\
Anion gap & 0.008 & & -0.202 & -1.755 & $-0.017-0.001$ & 0.085 \\
NLR & 0.033 & & 0.439 & 3.866 & $0.050-0.016$ & 0.000 \\
\hline NLR: Neutrophil-lymphocyte ratio, SIRS: Systemic inflammatory response syndrome, BMI: Body mass \\
index.
\end{tabular}

\section{Discussion}

The results of this study indicate age variable did not significantly related with SIRS. The percentage of SIRS events between patients with age $\geq 36$ years and $<36$ years is almost similar $(51.1 \%$ and $48.9 \%)$. Observational analytic research with a cross-sectional design carried out by Nurdani et al. in his research on the ratio of neutrophils-lymphocyte and procalcitonin in sepsis patients shows that in sepsis patients aged 18-60 years, there is a strong and significant correlation between lymphocyte-neutrophils ratios and procalcitonin levels in sepsis patients. Gender in this study was obtained not related to the occurrence of SIRS. Male patient occupying the most occurring SIRS

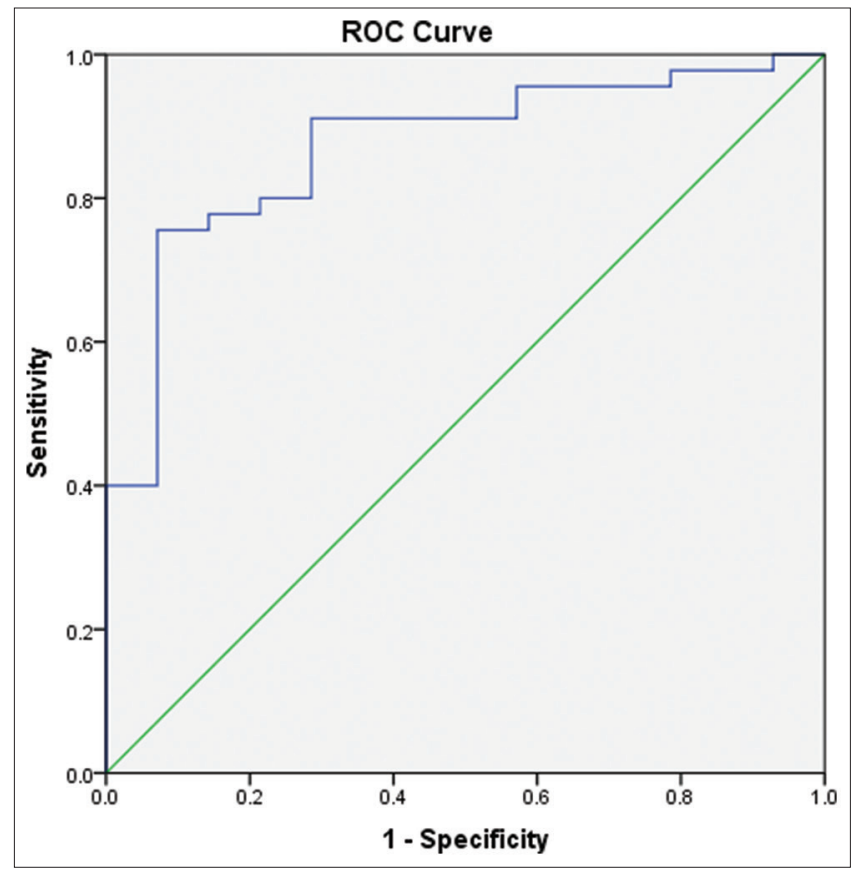

Figure 1: Neutrophil-lymphocyte ratio sensitivity based on receiver operating characteristic curve 
(71.1\%). IMT in this study also showed results that were not related to the occurrence of SIRS. SIRS more occurring in patient with normal BMI [4].

Blood sugar levels are related to the occurrence of SIRS. Initial hyperglycemia is associated with increased mortality (37\% in hyperglycemia groups with $8 \%$ in the normoglycemia group). This shows the close relationship between trauma and glucose levels. In research conducted by Bar-Or et al. in his research on stress hyperglycemia in critically ill patients mentioned more than $200 \mathrm{mg} / \mathrm{dL}$, blood sugar levels significantly increased the risk of severe infections and mortality in trauma patients. It is stated that hyperglycemia causes a decrease in response to infection with decreasing neutrophil chemotaxis, bond with endothelial, phagocytosis, and cellular immunity response.

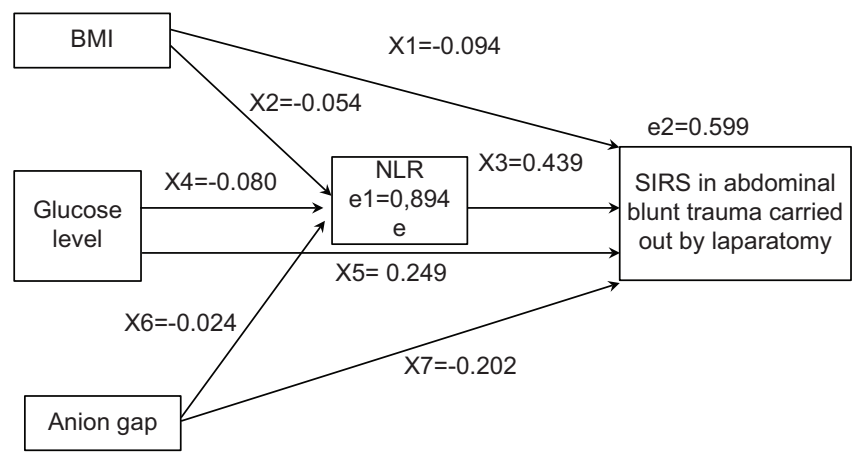

Figure 2: Path analysis

Research conducted by Van den Berghe et al. about insulin therapy in critical patients found that tight glucose control ( $\leq 110 \mathrm{mg} / \mathrm{dL}$ ) caused a significant decrease in the overall death rate and a decrease in complications associated with prolonged treatment in intensive room such as kidney failure, time on ventilator, and infection rate. Starting therapy with insulin can play an important role in inflammatory response. The study shows that both insulin and glucose can affect systemic inflammatory responses. Giving glucose and insulin increase the inflammatory response to dangerous stimulus. Hyperinsulinemia indirectly strengthens the component of inflammatory and stress responses to infection. Hyperglycemia can also deactivate immunoglobulin and contribute to the risk of infection [5], [6], [7], [8].Based on the picture above, we can conclude that NLR has a higher direct effect on SIRS (43.9\%) than other the variable (BMI, glucose, and anion gap). Multiple trauma is a significant cause of patient morbidity and mortality, and is the most common cause of death in young patients. Trauma remains the main cause of death in the world in patients with age under 45 years. Hypoxia, hypovolemia, and serious organ injury are direct and indirect causes of death due to trauma in the first few hours after injury. Homeostasis system tries to limit injury causing activation of immune cell function, release of local and systemic anti-inflammatory mediators, coagulation cascade activation, and complement system. After a severe trauma, there is an excessive activation of the immunological response which then develops into SIRS. The initial pro-inflammatory response was followed by anti-inflammatory response syndrome, which caused immune emphasizing and increase risk development of severe infections and sepsis. Sepsis is the most common cause of death in the final phase of trauma [9], [10].

Anion gap is related to the SIRS incident. Anion gap $\geq 12$ is obtained by SIRS by $77.8 \%$. In a study conducted by Mohr et al, regarding usage of anion gap serum to identify risk of death in sepsis patients, stated that blood lactic level more than $4 \mathrm{mmol} / \mathrm{L}$ increase mortality risk significantly, and lactate clearance towards normal level proved to be significant in reducing mortality risks. Furthermore, increased attention has focused on lactate measurement earlier, due to lactic $>2.0 \mathrm{mmol} / \mathrm{L}$ which has been defined as inclusion criteria for initial management bundles sepsis Sep-1 who passed the CMS. Lactate is a common cause of anion gap metabolic acidosis and increased lactate associated with an increase in anion gaps and a decrease in serum bicarbonate. According to that study, anion gap and serum bicarbonate were not good at predicting lactic changes and mortality. In limited resource settings where lactate is not available, anion gap $\geq 20 \mathrm{mEq} / \mathrm{L}$ may be used for the patient's risk stratification so that sepsis treatment can be given, but lactate is also chosen as sepsis biomarkers. In a research conducted with Berkman et al. regarding anion gap as a screening tool for elevated lactate level in patients with an increased risk of developing sepsis in the emergency department, stated that an increase in anion gap (>12 $\mathrm{mmol} / \mathrm{L})$ in predicting increased lactic levels ( $>4 \mathrm{mmol} / \mathrm{L})$ had sensitivity value of $80 \%(72-87 \%)$ with specificity value $69 \%(66-71 \%)$. Patients with anion gap $>12 \mathrm{mmol} / \mathrm{L}$ have a 7.3 -fold risk $(95 \% \mathrm{Cl} 4.6-11.4)$ in resulting an increased lactic level above $4 \mathrm{mmol} / \mathrm{L}$ [11], [12].

The sensitivity of the NLR on the SIRS incidence was high enough $92.3 \%$ with a specificity of $55 \%$, a positive predictive value of $80 \%$, and a negative predictive value of $78 \%$ with an accuracy of $79.6 \%$. Different results in the population of children in Mathews et al. in 2019 found the accuracy of NLR and platelet-lymphocyte ratio amounted to $72.73 \%$ in predicting mortality. The ratio of NLR is considered a cheap, simple, and promising parameter to predict the severity of sepsis and NLR can be used as a parameter to predict the severity of sepsis in hospitals with limited laboratory facilities [13], [14].

Research by Darwis and Probosusena concerning the relationship between the NLR and outcomes of sepsis on geriatric patients had different results from our study, which analyzed the sensitivity and specificity of the NLR value to determine the death of sepsis on the geriatric patients. According to Darwis and Probosusena research, based from ROC curve, NLR had a good predictor value (AUC 66.6\%; p = 0.04) with 13.05 as their cutoff point. Eighteen geriatric patients $(66.67 \%)$ with sepsis which had NLR value $>13.05$ were 
dead, compared with 9 geriatric patients $(36 \%)$ with NLR values $<13.05$. Based on this result, it can be concluded that an increase in NLR is associated with increased mortality in geriatric patients with sepsis [15].

In a study conducted by Kaushik et al in 2018, studying NLR diagnostic and prognostic role in early and late phase of sepsis patients had involved 56 patients of newly diagnosed sepsis cases and 20 healthy adults as control. This study results concluded that NLR has a promising role as a diagnostic and prognostic marker (with $p=0.001$ and $p=0.045$, respectively) for sepsis. From this study conclusion, it can be underlined that NLR can be used as diagnostic and prognostic marker for sepsis patients [16].

Martins et al. in 2019 had studied the role of NLR for patients with early sepsis on treatment in intensive room. The value of AUC from ROC curve was 0.62 for NLR, 0.98 for neutrophils, and 0.51 for total leukocytes. NLR value above 5.0, leukocytes count above 12,000 $\mathrm{mm}^{3} / \mathrm{ml}$, and the percentage of neutrophil band above $10 \%$ were contributed as risk factors for sepsis. Lymphocyte and neutrophil band ratios on combination with other parameters can be used as a marker for early detection in sepsis patients residing in ICU [17].

NLR is the most dominant risk factor in the study compared with age, BMI, blood sugar levels, anion gap, and trauma status in blunt abdominal trauma patients carried out by laparotomy. The results of the study from Liu et al. about the significance prognosis of the NLR in sepsis patients found a significantly higher level of median NLR in patients who died from survivors. NLR has a simple power to predict poor results as suggested by the AUC $0.695 \pm 0.036$. Multivariate linear regression showed elevated levels of NLR associated with unfavorable outcomes independently of confounding effects. Spearman's correlation test shows that there is a positive correlation between the NLR levels and the severity of the disease [18].

$\mathrm{Ni}$ et al. research on the ratio of neutrophilslymphocytes as a prognosis marker in sepsis patients who died in the hospital stated the ratio of the NLR as a prognostic marker in inflammatory disease. Contrary to this study, the larger NLR was found to have fewer chances of death in hospitals as well as the existence of bacteremia. Patients who have shock or have a history of chronic heart failure have a greater chance of mortality while in hospitals. The multivariate logistic regression analysis shows that low NLR is the independent predictor of death in hospitals $(\mathrm{OR}=-0.98 ; 95 \% \mathrm{Cl}: 0.96-0.99 ; \mathrm{p}=0.022)$. However, there were no correlations found between NLR and 28-day hospital mortality in patients with sepsis $(p=0.988)$. As a predictor of survival in the hospital, the area under the NLR curve (AUC) is 0.622 (95\% Cl $0.54-0.71 ; p=0.006)$ and the cutoff point value is 9.11 with a sensitivity of 0.551 and 0.707 specific, NLR when admitted to hospital is a predictor of independent mortality of sepsis patients in the hospital [19].
Various studies assume surgery/trauma resulted in pro-inflammatory conditions (SIRS). However, it has been well known that surgery and trauma cause selective suppression of Th1 function, improve the function as a suppressor of Th2 cellular immune response. The decline of Th1 level can increase the risk of infection, such as pneumonia, wound infection, and septicemia. Many reports in the past two decades indicate that there will be a change in proliferation response to cell mitogens immediately after trauma or thermal injury. T-cell dysfunction after trauma is characterized by a decrease in T-cell proliferation, cytokine profiles distorted, decrease the interaction of monocytes and $\mathrm{T}$ cells, and weakening the expression of T-cell receptor complex. Likewise, surgery and trauma cause balance disorders Th1/Th2 causes disturbances in cell-mediated immunity [20], [21], [22].

To the best of our knowledge, this is the first study from Bali reviewing the effects of elevated NLR toward SIRS in trauma patients. This study not only reviewing risk factors regarding SIRS in trauma patients but also extending their roles contributed to SIRS by multivariate analysis, with NLR as the most significant variables. There are some limitations noted from this study. First, this study is an observational prospective design with SIRS as its outcome, without further follow-up examination. This issue causes incomplete data regarding complication and mortality following SIRS due to multitrauma. Further study regarding morbidity and prognostic data in multitrauma patients with elevated NLR is needed in the future. Second, this study lacks additional data regarding patient's basic information, such as patient's specific injury, mechanism of injury, prehospital care, and surgery required in these patients. These variables could not be taken completely because some of these data were not completely recorded in our system. Third, this study was using BMl which was divided into two criteria (abnormal $<18$ or $>25$ and normal BMI). Due to this category, we could not asses either underweight or overweight patients, especially their clinical outcome after emergency laparotomy.

\section{Conclusion}

Blood glucose levels, high anion gap, and multitrauma status are associated with the occurrence of SIRS in blunt abdominal trauma patients carried out by laparotomy. NLR is a predictor of SIRS in blunt abdominal trauma carried out by emergency laparotomy, can also be used as a diagnostic tool to screen the incidence of SIRS. The lymphocyte-toneutrophil ratio has a high sensitivity of $92.3 \%$ with an accuracy of $79.6 \%$ with the occurrence of SIRS. NLR is a dominant risk factor compared to other factors tested 
(43.9\%) on the occurrence of SIRS in blunt abdominal trauma patients carried out by laparotomy. The NLR can be used as a prognostic marker on the incidence of SIRS, especially in peripheral hospitals with the limitations of other infection marker examination methods.

\section{References}

1. El-Menyar A, Abdelrahman $\mathrm{H}, \mathrm{Al}$-Thani $\mathrm{H}$, Zarour A, Parchani $\mathrm{A}$ Peralta $\mathrm{R}$, et al. Compartmental anatomical classification of traumatic abdominal injuries from the academic point of view and its potential clinical implication. J Trauma Manag Outcomes. 2014;8:14. https://doi.org/10.1186/1752-2897-8-14 PMid:25332723

2. Hazeldine J, Hampson P, Lord JM. The impact of trauma on neutrophil function. Injury. 2014;45(12):1824-33. https://doi. org/10.1016/j.injury.2014.06.021

PMid:25106876

3. Koirala U, Thapa PB, Joshi MR, Singh DR, Sharma SK. Systemic inflammatory response syndrome following gastrointestinal surgery. JNMA J Nepal Med Assoc. 2017;56(206):221-5. https:// doi.org/10.31729/jnma.3144

PMid:28746319

4. Nurdani A, Hadi U, Arfijanto M, Rusli M, Bramantono B, Miftahussurur M. Neutrophil-lymphocyte ratio and procalcitonin levels in sepsis patients. N Am Med J. 2019;13:48-54.

5. Shi J, Dong B, Mao Y, Guan W, Cao J, Zhu R, et al. Review: Traumatic brain injury and hyperglycemia, a potentially modifiable risk factor. Oncotarget. 2016;7(43):71052-61. https:// doi.org/10.18632/oncotarget.11958

PMid:27626493

6. Bar-Or D, Rael LT, Madayag RM, Banton KL, Tanner A Acuna DL, et al. Stress hyperglycemia in critically III patients: Insight into possible molecular pathways. Front Med (Lausanne). 2019;6:54. https://doi.org/10.3389/fmed.2019.00054 PMid:30972338

7. Soop M, Duxbury H, Agwunobi AO, Gibson JM, Hopkins SJ, Childs $\mathrm{C}$, et al. Euglycemic hyperinsulinemia augments the cytokine and endocrine responses to endotoxin in humans. Am J Physiol Endocrinol Metab. 2002;282(6):E1276-85. https://doi. org/10.1152/ajpendo.00535.2001

PMid:12006357

8. van den Berghe $G$, Wouters $P$, Weekers $F$, Verwaest $C$, Bruyninckx $F$, Schetz $M$, et al. Intensive insulin therapy in critically ill patients. N Engl J Med. 2001;345(19):1359-67. https://doi.org/10.1056/nejmoa011300

PMid:11794168

9. Arumugam $\mathrm{S}$, Al-Hassani $\mathrm{A}$, El-Menyar $\mathrm{A}$, Abdelrahman $\mathrm{H}$, Parchani A, Peralta R, et al. Frequency, causes and pattern of abdominal trauma: A 4-year descriptive analysis. J Emerg Trauma Shock. 2015;8(4):193-8. https://doi. org/10.4103/0974-2700.166590 PMid:26604524

10. Trancă SD, Petrişor CL, Hagău N. Biomarkers in polytrauma induced systemic inflammatory response syndrome and sepsis-a narrative review. Rom J Anaesth Intensive Care. 2014;21(2):118-22

Author Queries???

AQ6: Please fix the grammatical errors

AQ7: Please apply this format for whole manuscript.
PMid:28913443

11. Mohr NM, Vakkalanka JP, Faine BA, Skow B, Harland KK, DickPerez R, et al. Serum anion gap predicts lactate poorly, but may be used to identify sepsis patients at risk for death: A cohort study. J Crit Care. 2018;44:223-8. https://doi.org/10.1016/j. jcrc.2017.10.043

PMid:29175046

12. Berkman M, Ufberg J, Nathanson LA, Shapiro NI. Anion gap as a screening tool for elevated lactate in patients with an increased risk of developing sepsis in the emergency department. J Emerg Med. 2009;36(4):391-4. https://doi.org/10.1016/j. jemermed.2007.12.020

PMid:18815002

13. Arif SK, Rukka ABS, Wahyuni S. Comparison of neutrophilslymphocytes ratio and procalcitonin parameters in sepsis patient treated in Intensive Care Unit Dr. Wahidin Hospital, Makassar, Indonesia. J Med Sci. 2017;17(1):17-21. https://doi. org/10.3923/jms.2017.17.21

14. Mathews S, Rajan A, Soans S. Prognostic value of rise in neutrophil to lymphocyte ratio (NLR) and platelet to lymphocyte ratio (PLR) in predicting the mortality in paediatric intensive care. Int J Contemp Pediatr. 2019;6(3):1052-8. https://doi. org/10.18203/2349-3291.ijcp20191044

15. Darwis I, Probosuseno P. The relationship between neutrophillymphocyte ratio and sepsis outcome in geriatric patients. JK Unila. 2019;3(1):147-53.

16. Kaushik R, Gupta M, Sharma M, Jash D, Jain N, Sinha N, et al. Diagnostic and prognostic role of neutrophil-to-lymphocyte ratio in early and late phase of sepsis. Indian J Crit Care Med. 2018;22(9):660-3. https://doi.org/10.4103/ijccm.ijccm_59_18 PMid:30294133

17. Martins EC, Da Fe Silveira L, Viegas $K$, Beck $A D$, Fioravantti G Jr., Cremonese RV, et al. Neutrophil-lymphocyte ratio in the early diagnosis of sepsis in an intensive care unit: A case-control study. Rev Bras Ter Intensiva. 2019;31(1):64-70. https://doi.org/10.5935/0103-507x.20190010 PMid:30916236

18. Liu X, Shen $Y$, Wang $H$, Ge Q, Fei A, Pan S. Prognostic significance of neutrophil-to-lymphocyte ratio in patients with sepsis: A prospective observational study. Mediators Inflamm. 2016;2016:8191254. https://doi.org/10.1155/2016/8191254 PMid:27110067

19. Ni J, Wang H, Li Y, Shu Y, Liu Y. Neutrophil to lymphocyte ratio (NLR) as a prognostic marker for in-hospital mortality of patients with sepsis: A secondary analysis based on a single-center, retrospective, cohort study. Medicine (Baltimore). 2019;98(46):e18029. https://doi.org/10.1097/ $\mathrm{md} .0000000000018029$

PMid:31725679

20. Marik PE, Flemmer M. The immune response to surgery and trauma: Implications for treatment. J Trauma Acute Care Surg. 2012;73(4):801-8. https://doi.org/10.1097/ta.0b013e318265cf87 PMid:22976420

21. Lord JM, Midwinter MJ, Chen YF, Belli A, Brohi K, Kovacs EJ, et al. The systemic immune response to trauma: An overview of pathophysiologyand treatment. Lancet. 2014;384(9952):1455-65. https://doi.org/10.1016/s0140-6736(14)60687-5 PMid:25390327

22. Menges P, Kessler W, Kloecker C, Feuerherd M, Gaubert S, Diedrich $\mathrm{S}$, et al. Surgical trauma and postoperative immune dysfunction. Eur Surg Res. 2012;48(4):180-6. https://doi. org/10.1159/000338196 PMid:22653168 